\title{
Ultrafast Response of Arrayed Waveguide Gratings
}

\author{
Luis Grave de Peralta, Ayrton A. Bernussi, and Henryk Temkin, Fellow, IEEE
}

\begin{abstract}
The ultrafast limit of the response of arrayed waveguide gratings is studied both theoretically and experimentally. We present new experimental results that show that interference occurs inside of an arrayed waveguide pulse shaper even when pulses that travel through different paths do not overlap. A comprehensive discussion of this extreme case is presented.
\end{abstract}

Index Terms-Gratings, interference, optical planar waveguide components, optical pulse shaping, ultrafast optics, waveguide arrays, wavelength-division multiplexing (WDM).

\section{INTRODUCTION}

A RRAYED waveguide gratings (AWGs) are extremely versatile integrated-optics devices [1], [2]. AWG multiplexers are the corner stone in currently deployed wavelength-division multiplexing (WDM) communication systems [3], [4]. Detailed theoretical methods have been developed for simulation of the spectral response of AWG multiplexers [5], [6]. Recently, direct space-time pulse shapers based on AWGs [7], [8] have been demonstrated. Pulse shapers (optical encoders) are key devices for future optical code-division multiple-access (CDMA) communication networks [9]. Even though an analogy between bulk space-time and AWG pulse shapers (AWG-PSs) has already been established [7] and simple design formulas for AWG-PS have been previously described [8], [10], [11], there is still a great need for specific methods for precise simulation of the temporal and spectral response of the AWG-PS. In this paper, we present a detailed model for the simulation of AWG-PS on a femtosecond time scale. In addition, we show new experimental results for the extreme case of AWG-PS operating in the nonoverlapping-pulses (NOP) regime.

The remainder of this paper is structured as follows. Section II contains a general quantitative description of the temporal and spectral response of AWGs to a single ultrashort optical input pulse. Two cases are distinguished depending on the occurrence of pulse overlapping inside of the device. Furthermore, it is shown that the quantitative model predicts the occurrence of interference inside of the AWG-PS in both cases. In Section III, we present novel experiments using an AWG-PS especially fabricated for testing the model prediction of interference inside of a pulse shaper operating in the NOP regime. A discussion of the experimental results is presented in Section IV. It is shown

Manuscript received October 3, 2006; revised December 15, 2006. This work was supported in part by the Jack F Maddox Foundation. Work at Multipass Corporation was supported by the SBIR-NSF under Grant 0450072

L. G. de Peralta is with the Multipass Corporation, Lubbock, TX 79401 USA (e-mail: luisgrave@ hotmail.com).

A. A. Bernussi and H. Temkin are with the Department of Electrical and Computer Engineering, Texas Tech University, Lubbock, TX 79410 USA (e-mail: ayrton.bernussi@ttu.edu; henryk.temkin@ttu.edu).

Digital Object Identifier 10.1109/JQE.2007.895659

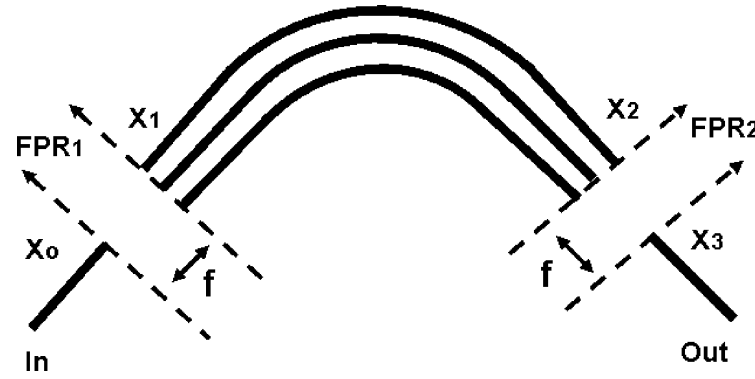

Fig. 1. Schematic representation of a conventional AWG layout. Only the central input and output waveguides are shown. Represented planes $\mathrm{x}_{o}, \mathrm{x}_{1}, \mathrm{x}_{2}$, and $\mathrm{x}_{3}$ are arcs of circles with radius of curvature $f$.

that the experimental results agree nicely with the predictions of our quantitative model. Finally, we conclude in Section V. This body of work represents the first comprehensive overview specifically dedicated to AWGs in the genuine ultrafast limit, i.e., under conditions where no overlapping of pulses occurs inside of the device, from both a theoretical as well as an experimental point of view. Application of the quantitative model to previously published experiments [8]-[10] where external modulation was applied will be published elsewhere.

\section{QUANTITATIVE DESCRIPTION}

The schematic of a conventional AWG layout is shown in Fig. 1. It consists of a set of input waveguides, followed by a slab or free propagation region (FPR), which is continued by an array of waveguides that acts as a grating, followed by a second FPR and ending with another set of output waveguides [4]. The length of each consecutive waveguide in the grating is incremented by a fixed amount $\Delta L$ [4]. We assume here that both FPRs have the same radius of curvature $(f)$ and that consecutive waveguides in the grating are separated by the distance $d$ at both FPRs extremes. The key planes to be investigated in the following are also shown in Fig. 1.

Consider an optical pulse of short duration leaving the central input waveguide ( $x_{o}=0$ ) with temporal profile $e_{\mathrm{in}}(t)$ and with its transversal spatial distribution profile equal to the waveguidemode transverse profile $w_{o}\left(x_{o}\right)$ [5] as

$$
e_{o}\left(x_{o}, t\right)=w\left(x_{o}\right) e_{\text {in }}(t)
$$

with

$$
e_{\text {in }}(t)=u(t) e^{j 2 \pi \nu_{o} t}
$$

where $u(t)$ is the slowly varying amplitude of the field and $v_{o}=$ $\omega_{o} / 2 \pi$ is the central frequency of the pulse spectrum $E_{\mathrm{in}}(\omega)$ defined as [7]

$$
E_{\text {in }}(\omega) \propto \int e_{\text {in }}(t) e^{-j \omega t} d t
$$


Here, we will assume transform-limited pulses of duration $\tau_{\mathrm{p}} \sim 1 / \Delta v$, where $\Delta v$ is the spectral width of the pulse spectrum. Due to the small size of the AWGs and the single-mode character of the waveguides, we will neglect any pulse deformation due to chromatic dispersion. In addition, we consider an AWG with an ideal geometry, i.e., with zero dispersion related to phase errors [12], [13]. The field $e_{o}(x, t)$ is radiated from the central input waveguide to the first FPR. The light spatial distribution $\left(e_{1-}\right)$ arriving to the focal plane $x_{1}$ of Fig. 1 can be obtained by the spatial Fourier transform of the input distribution [5], whereas the temporal profile at the slab-grating interface is identical to that of the input pulse except for a phase factor, thus, the field arriving to the grating is described by

$$
e_{1-}\left(x_{1}, \omega\right) \propto W\left(x_{1}\right) E_{\text {in }}(\omega)
$$

with

$$
\begin{aligned}
W\left(x_{1}\right) & \propto F_{x_{1}}\left[w\left(x_{1}\right)\right]_{u=x_{1} / \alpha} \\
\alpha & =\frac{\lambda_{o} f}{n_{\mathrm{s}}} \\
\lambda_{o} & =\frac{c}{\nu_{o}}
\end{aligned}
$$

where $F_{x}$ denotes the spatial Fourier transform, $c$ is the speed of the light, and $n_{\mathrm{s}}$ is the effective refractive index of the slab region. The light coupled into each of the $N+1$ waveguides of the grating is proportional to the overlap integral between the spatial distribution of the illuminating field $W\left(x_{1}\right)$ and the waveguide mode $w\left(x_{1}\right)$ [5]. Therefore, the field coupled to the grating is given by

$$
e_{1}\left(x_{1}, t\right) \propto \int s\left(x_{1}\right) E_{\mathrm{in}}(\omega) e^{j \omega t} d \omega
$$

with

$$
s\left(x_{1}\right)=\sum_{k=-N / 2}^{N / 2} c_{k} W(k d) w\left(x_{1}-k d\right)
$$

The parameter $c_{k}$ was included in (1) to account for external modulation of the grating. Phase or amplitude modulation can be implemented experimentally by using a combination of a reflective AWG and a spatial light modulator used as the external reflector [8], [10], [11]. Here, we will focus the attention in the case $c_{k}=1$, which corresponds to absence of external modulation. Using (2), (1) can be rewritten as

$$
e_{1}\left(x_{1}, t\right) \propto \sum_{k=-N / 2}^{N / 2} c_{k} W(k d) w\left(x_{1}-k d\right) \int E_{\mathrm{in}}(\omega) e^{j \omega t} d \omega
$$

Equation (3) without external modulation $\left(c_{k} \equiv 1\right)$ shows that the total field coupled to the grating can be described as $N+1$ low-intensity pulse replicas simultaneously coupled to different waveguides of the grating and each having the same spectrum of the input pulse.

By design, there is a constant length difference $\Delta \mathrm{L}$ between consecutive waveguides in the grating [4]

$$
\Delta L=m \frac{\lambda_{o}}{n_{\mathrm{eff}}}
$$

where $n_{\mathrm{eff}}$ is the effective refractive index of the waveguides and $m$ is the diffraction order. This constant length difference results in a time delay inverse proportional to the free spectral range (FSR) of the device [7]

$$
\Delta \tau=\frac{\Delta L}{\frac{c}{n_{\mathrm{eff}}}}=\frac{1}{F S R}
$$

The spectral component with frequency $v=\omega / 2 \pi$ of the pulse replica traversing the $k$ th waveguide $(k=$ integer part of $x_{2} / d$ ) experiences a relative phase shift, with respect to the component with frequency $v_{o}$ of the pulse replica traversing the central waveguide in the grating, which is equal to [5]

$$
\Delta \phi\left(x_{2}, \omega\right)=e^{-j m\left(\omega / \nu_{o}\right)\left(x_{2} / d\right)}=e^{-j \omega\left((1 / F S R)\left(x_{2} / d\right)\right)} .
$$

Thus, the field in the other extreme of the grating (plane $x_{2}$ in Fig. 1) is given by the following expression:

$$
\begin{aligned}
e_{2}\left(x_{2}, t\right) \propto & \sum_{k=-N / 2}^{N / 2} c_{k} W(k d) w\left(x_{2}-k d\right) \\
& \times \int e^{-j \omega\left((1 / F S R)\left(x_{2} / d\right)\right)} E_{\mathrm{in}}(\omega) e^{j \omega t} d \omega .
\end{aligned}
$$

Equation (4), with $c_{k} \equiv 1$, shows that the total field leaving the grating can be described as $N+1$ low-intensity pulse replicas, each one coupled to a different waveguide of the grating. The pulse replicas have the same spectrum of the input pulse, but there is a delay $\Delta \tau=1 / \mathrm{FSR}$ between pulse replicas traversing consecutive waveguides of the grating. If the spectral width $\Delta v$ of $E_{\text {in }}(\omega)$ was smaller than the FSR $\left(\Delta \tau<\tau_{\mathrm{p}}\right)$, the pulse replicas would overlap at the focal point of the second FPR. In the opposite case, $\Delta v>\mathrm{FSR}$, the pulse length would be smaller than the separation between consecutive pulse replicas and no pulse overlapping could occur inside of the AWG. Equation (4) can be rewritten as follows:

$$
\begin{aligned}
e_{2}\left(x_{2}, t\right) \propto & \int d \omega \sum_{k=-N / 2}^{N / 2} c_{k} W(k d) w\left(x_{2}-k d\right) \\
& \times e^{-j \omega\left((1 / F S R)\left(x_{2} / d\right)\right)} E_{\mathrm{in}}(\omega) e^{j \omega t} .
\end{aligned}
$$

Equation (5) gives an alternative way to describe the total field leaving the grating. A Dirac's translational state [14] with frequency $v=\omega / 2 \pi$ leaves each waveguide of the grating. Ideal translational states are monochromatic and, thus, infinitely long. Actually, the length of real translational states is ultimately determined by the coherence length of the numerous modes lasing simultaneously in the cavity of the mode-locked laser often used as the pulsed light source [15]. This length is typically much larger than the AWG dimensions; consequently, we should expect that the $N+1$ translational states corresponding to the same frequency but leaving different waveguides of the grating overlap at the focal point of the second FPR. This would result in interference occurring inside of the AWG even in the absence of pulse overlapping. For comparison with the theoretical work 
in [7], it is helpful to rewrite (5) in terms of the masking function $s(x)$ defined by (2) as

$$
e_{2}\left(x_{2}, t\right) \propto \int E_{2}\left(x_{2}, \omega\right) e^{j \omega t} d \omega
$$

with

$$
\begin{gathered}
E_{2}\left(x_{2}, \omega\right) \propto s\left(x_{2}\right) e^{-j \gamma \omega x_{2}} E_{\text {in }}(\omega), \\
\gamma=\frac{1}{d F S R}=\frac{\Delta \tau}{d} .
\end{gathered}
$$

Thus, the field $\left(e_{3-}\right)$ arriving at the output waveguides (plane $x_{3}$ in Fig. 1) is the spatial Fourier transform of (6) with the result [7]

$$
e_{3-}\left(x_{3}, t\right) \propto \int S\left(\frac{2 \pi x_{3}}{\alpha}-\frac{\Delta \tau}{d} \omega\right) E_{\text {in }}(\omega) e^{j \omega t} d \omega
$$

with

$$
S\left(\frac{2 \pi x_{3}}{\alpha}-\frac{\Delta \tau}{d} \omega\right) \propto F_{x_{2}}\left[s\left(x_{2}\right) e^{-j \gamma \omega x_{2}}\right]_{u=x_{3} / \alpha} .
$$

Note that the complex spectrum of the field arriving to the plane $\mathrm{x}_{3}$ is given by

$$
E_{3-}\left(x_{3}, \omega\right) \propto S\left(\frac{2 \pi x_{3}}{\alpha}-\frac{\Delta \tau}{d} \omega\right) E_{\text {in }}(\omega)
$$

and $S(\ldots)$ is the spectral response function of the AWG. Now consider a thin slit at the lateral position $\mathrm{x}_{3}=\mathrm{x}_{\mathrm{s}}$, i.e., of the form $\delta\left(\mathrm{x}_{3}-\mathrm{x}_{\mathrm{s}}\right)$. The filtered spectrum now has the form

$$
E_{3}\left(x_{3}, \omega\right) \propto S\left(\frac{2 \pi x_{3}}{\alpha}-\frac{\Delta \tau}{d} \omega\right) E_{\text {in }}(\omega) \delta\left(x_{3}-x_{\mathrm{s}}\right) .
$$

Equation (7) shows that a transverse movement of the output slit leads to a simple shift in the spectral response of the AWG, i.e., different frequencies leaves different output waveguides of the AWG. This occurs independently of the spectral with of the input pulse, i.e., as expected, interference occurs inside of the AWG even in the extreme case that there is no pulse overlapping inside of the device. With the slit at the central output waveguide $\left(x_{\mathrm{s}}=0\right)$, the field after the slit is then given by

$$
e_{3}\left(x_{3}=0, t\right) \propto \int S\left(-\frac{\Delta \tau}{d} \omega\right) E_{\text {in }}(\omega) e^{j \omega t} d \omega .
$$

As (8) shows, the temporal profile of the field coupled to the output slit is determined by the temporal profile of the input field convolved with a scaled version of the masking function

$$
e_{\text {out }}(t) \propto e_{\text {in }}(t) * s\left(-d \frac{t}{\Delta \tau}\right) .
$$

$s(-d t / \Delta \tau)$ has maxima at multiple values of $\Delta \tau$. Without external modulation, if the spectral width of the input pulse spectrum is smaller than the $\operatorname{FSR}\left(\Delta \tau<\tau_{\mathrm{p}}\right)$, the convolution (9) results in an elongated output pulse. This corresponds to an output spectrum containing a single narrow peak. In the opposite case, $\Delta v>\mathrm{FSR}$, the pulsewidth is smaller than the period of the scaled version of the masking function. This results in a

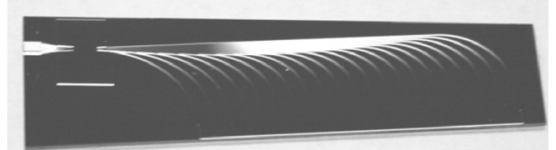

(a)

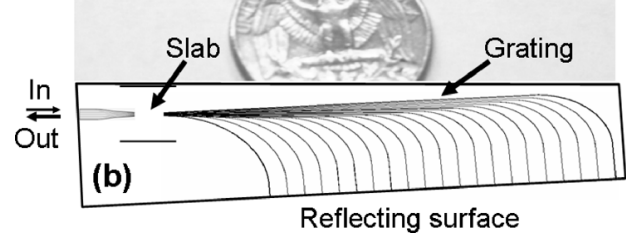

Fig. 2. (a) Photograph and (b) schematics of the fabricated AWG-PS.

burst of pulses with a repetition rate equal to $\Delta \tau$ and a multiple-peaked spectrum with a peak-to-peak separation equal to the FSR.

\section{EXPERIMENTS}

The AWG-PS used in this study was especially fabricated for testing the quantitative model prediction of interference inside of a pulse shaper operating in the NOP regime. It includes a waveguide-based grating fabricated using silica-on-silicon planar lightwave technology and arranged in a reflective geometry [16]. A photograph and a schematic presentation of the reflective AWG layout are shown in Fig. 2.

The AWG-PS consists of an input-output coupler, a 3-mm-long slab region, an arrayed waveguide grating with 21 waveguides, and a reflecting surface. High reflectivity at the reflecting surface was assured by the deposition of a $\mathrm{Cr}-\mathrm{Au}$ film. The waveguides forming the grating have a constant length increment of $2 \mathrm{~mm}$. Separation between waveguides is larger than $25 \mu \mathrm{m}$, which prevents any light coupling between them. The input-output structure contains one input and six output waveguides, with all waveguides placed perpendicular to the coupler-slab interface. For optical measurements, an array of optical fibers (not shown in Fig. 2) is attached to the input-output coupler of the AWG-PS. The device was designed to operate at a center wavelength of $1.56 \mu \mathrm{m}$ with a channel-to-channel frequency separation of $25 \mathrm{GHz}$ and a free spectral range of $50 \mathrm{GHz}$. Each waveguide in the device has a cross section of $5 \times 5 \mu \mathrm{m}^{2}$, and the step refractive index difference between core and cladding is $\sim 0.01$.

In the spectral and temporal experiments described below, a passive mode-locked laser (Femtomaster from Fianium) was used to illuminate the AWG-PS through its input waveguide. The laser, with an average power of $5 \mathrm{~mW}$, emits 0.5 -ps pulses with a repetition rate of $50 \mathrm{MHz}$. The laser has a time-limited spectrum with a spectral width of $\sim 7 \mathrm{~nm}$. An optical spectrum analyzer with $0.1-\mathrm{nm}$ resolution was used to record the power spectra at the output of the laser and at the output waveguides (output channels) of the device. The temporal profile of the output signals were recorded via intensity auto-correlation in a free-space apparatus.

We measured the spectral and temporal output response of a single output channel of the AWG-PS, averaged over many 


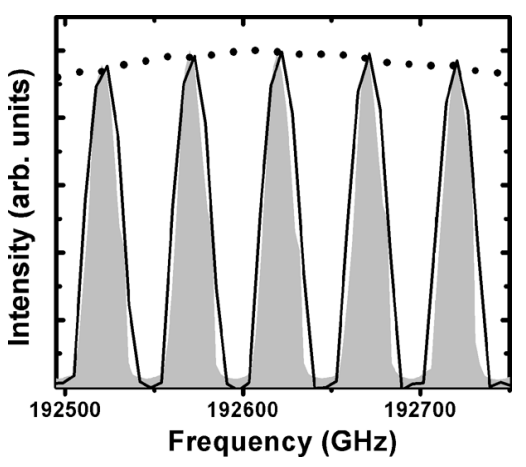

Fig. 3. Measured (filled curve) and calculated spectra (continuous line) corresponding to a single output channel of the AWG-PS. The spectrum of the mode-locked laser $(\bullet)$ is shown as a reference.

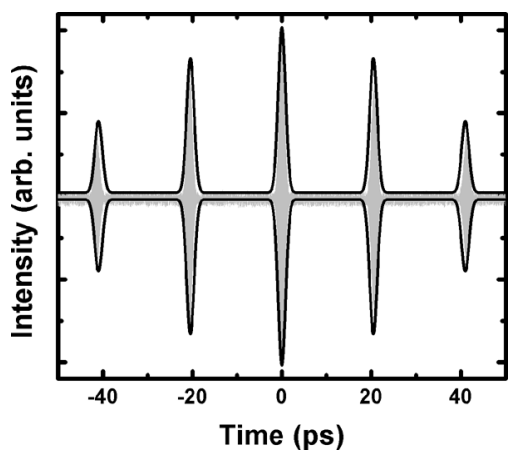

Fig. 4. Measured (filled curve) and calculated (continuous line) intensity autocorrelation traces corresponding to a single output channel of the AWG-PS.

input pulses. The single-channel output spectrum and the corresponding intensity autocorrelation trace are shown in Figs. 3 and 4 , respectively. The spectrum of the mode-locked laser is included as a reference.

The single-channel output spectrum consists of numerous narrow peaks separated by $\Delta \varepsilon \sim 50 \mathrm{GHz}$. The intensity distribution of the peaks is modulated by the spectral envelope of the mode-locked laser (Fig. 3). The intensity autocorrelation trace exhibits a train of pulses with the pulse-to-pulse separation of $\Delta \tau=1 / \Delta \varepsilon \sim 20$ ps (Fig. 4), as expected from the time delay imposed by the length increment between individual waveguides of the grating.

We also compare the output response of the AWG-PS measured at two different output channels. Details of the measured output spectra for two consecutive output waveguides are shown in Fig. 5. A relative shift of $\sim 25 \mathrm{GHz}$ is clearly observed comparing the two spectra.

\section{Discussion}

In the AWG-PS described here, the incident pulse diffracts out of the input waveguide and, after propagation through the slab region, illuminates the grating launching a pulse replica in each waveguide of the grating [see (3)]. The pulse replicas have lower intensity but the same spectrum as the input pulse. After returning from the reflecting surface, each pulse replica diffracts out of a waveguide of the grating [see (4)] and, after propagating back through the slab region, illuminates the input-output coupler launching an output pulse in each output waveguide. As

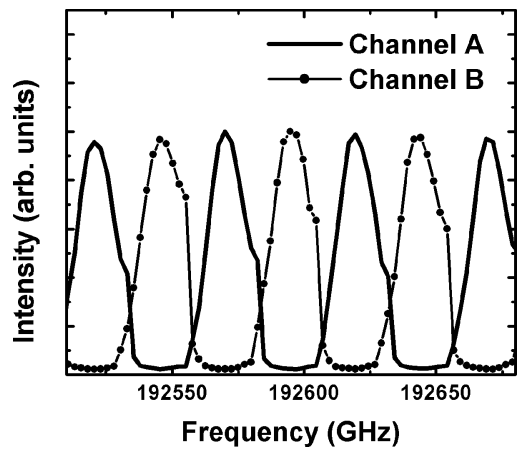

Fig. 5. Details of the spectra measured at two different output channels of the AWG-PS (channels A and B).

a result, when the AWG-PS is illuminated with a single input pulse, the temporal response at each output channel of the device is a burst of pulses [see (9) in Section II for the case $\Delta v>$ FSR]. The burst last several hundred picoseconds, which is much longer than the width of the pulses in the burst but much shorter than the 20-ns separation between bursts produced by consecutive input pulses. It is worth noticing that the spatial extent of the 0.5-ps pulse replicas in silica glass is $\sim 104 \mu \mathrm{m}$, and the round-trip pulse replica-to-pulse replica separation is $4 \mathrm{~mm}$. This is $1 \mathrm{~mm}$ longer than the length of the slab region. Therefore, the output pulses related with a pulse replica are already traveling through the output waveguides while the next pulse replica is still traversing the grating. Thus, there is never more than one pulse replica in the slab region which is the only place inside of the AWG-PS where superposition of pulse replicas could occur. This claim is supported by the results shown in Fig. 4. The arrayed waveguide grating acts as a series of delay lines, where each pulse in the output train is associated with a specific waveguide of the grating. The delay increment of $\sim 20 \mathrm{ps}$ between pulse replicas traversing consecutive waveguides is much larger than the width of the input pulse. Thus, there is no temporal and spatial overlap between pulse replicas, at any moment, inside of the AWG-PS. In addition, there is no overlap between pulses in the output burst, as clearly shown by the autocorrelation trace in Fig. 4.

The multiple-peaked nature of the spectrum of an output channel is expected from a simple Fourier analysis. As expected, a modulated temporal trace with period of $\Delta \tau \sim 20 \mathrm{ps}$ (Fig. 4) results in a periodic multiple-peaked spectrum with frequency separation of $\Delta \varepsilon=1 / \Delta \tau \sim 50 \mathrm{GHz}$ (Fig. 3). This is equal to the design value of the device free spectral range. A frequent physical description for the results shown in Fig. 3 asserts that pulses in the output train become stretched by the external optical analyzer used to measure the spectrum. Stretched pulses overlap inside the analyzer resulting in the so-called spectral interference [17], [18]. Based on this analysis, the observed interference (modulated spectrum shown in Fig. 3) would be attributed to the superposition of stretched pulses outside of the AWG-PS. However, the comparison between the output spectra from two different channels of the AWG-PS demonstrates that interference also occurs inside of the device, without any overlap between pulse replicas, and before the light arrives to the external apparatus used to measure the output spectrum. 
A clear frequency shift of $\sim 25 \mathrm{GHz}$ is observed in Fig. 5 comparing the output spectra measured at two consecutive channels of the AWG-PS. This is equal to the design value of the device channel-to-channel frequency separation, even in the ultrafast limit; light with different frequencies leaves different output channels of the AWG-PS.

The observed frequency shift is a characteristic signature of interference in multiple-slits experiments. Interference supposes superposition but the things that superpose inside of the extreme AWG-PS used in this work are not pulses. Using Fourier optics approaches (see Section II), each femtosecond pulse replica can be described as a superposition of monochromatic components [19] that satisfy two conditions: a large spectral bandwidth and each spectral component must have a specific phase relation to the other components [20]. Pulses are spatially confined in relatively small regions but, as discussed above [see (5)], the monochromatic Dirac's translational states are much longer. The Dirac's translational state with frequency $v=\omega / 2 \pi$, which traverses the $k$ th waveguide of the grating, is described by the following expression:

$$
\begin{aligned}
& f_{k, \omega}\left(x_{2}, t\right) \propto c_{k} W(k d) w\left(x_{2}-k d\right) e^{-j \omega\left((1 / \mathrm{FSR})\left(x_{2} / d\right)\right)} \\
& \times E_{\mathrm{in}}(\omega) e^{j \omega t}
\end{aligned}
$$

Note that ideal translational states are monochromatic and, thus, infinitely long. There are $N+1$ translational states with the same frequency traversing the grating simultaneously where each one traverses a different waveguide of the grating. Superposition of these $N+1$ extended translational states, corresponding to pulse replicas traversing different waveguides of the grating, produces that light with different frequencies leaves different output channels. The absence of temporal and spatial overlap between pulse replicas does not matter; as long as the Dirac's translational states corresponding to pulse replicas traversing different waveguides of the grating overlap coherently inside of the AWG-PS, interference occurs inside of the pulse shaper and the arrayed waveguide grating continues to yield precisely the spectral information expected of it.

Even when individual photons are not detected in the experiments described in this study, it is interesting to look at the experimental data shown in Figs. 3 and 4 from the more general point of view of quantum optics. In this approach, interference occurs because there are indistinguishable ways for an event to occur [21]. Here, the event is the detection of a photon which entered the AWG-PS at a defined time and left it with a specific delay through a particular output waveguide. The AWG device may be considered a multiple-slit arrangement, thus, a photon can arrive to the same output waveguide following numerous optical paths. Monitoring the burst of pulses at a single output waveguide (Fig. 4) makes (at least in principle) it possible to identify which waveguide of the grating a particular photon passed through. However, interference still occurs inside of the AWG-PS because it is not possible to simultaneously know the energy of the photon since the output spectrum is multiple-peaked (Fig. 3). If, intending to determine the energy of the photon, an external optical spectrum analyzer were coupled to the output channel, pulses of the output burst would be stretched in the external apparatus, resulting in a single elongated pulse. This would erase the which-path information. Absence of which-path information would be the reason why interference occurs in the combined system formed by the AWG-PS plus the external apparatus.

We provide a comparison between experimental and numerical results using the analytical formulas described in Section II. A Gaussian approximation was adopted for the mode transverse profiles of the waveguides, but all spatial and temporal Fourier transforms were calculated numerically using a fast Fourier transform (FFT) algorithm. First, single-channel output spectra were numerically calculated. Then, the temporal response of the device was evaluated as the temporal Fourier transform of the spectral response. Excellent agreement between calculated and measured spectra (autocorrelation traces) for a single output channel of the device can be clearly observed in Fig. 3 (Fig. 4), demonstrating the effectiveness of proposed model.

\section{CONCLUSION}

We have presented a general quantitative description of the temporal and spectral response of AWGs to a single ultrashort optical pulse. This model permits two complementary but equivalent descriptions of what happens in AWG-PSs. One description refers to propagation of a set of replicas of the input pulse. This description is more useful in the case that the spectral width of the pulse spectrum is smaller than the FSR of the device. In this case, the simplest description of the interference inside of the AWG-PS may be done in terms of superposition of overlapping pulse replicas at the focal point of the second FPR of the device.

The alternative description is more useful when the spectral width of the pulse spectrum is larger than the FSR of the device. In this case, pulse replicas traversing different waveguides of the grating do not overlap. However, we demonstrated that, even when pulses do not overlap at all inside of the device, it is still possible to describe the interference inside of the AWG-PS in terms of superposition of overlapping Dirac's translational states. In addition, we presented novel experiments using an AWG-PS especially fabricated for testing this extreme. The experimental results agreed nicely with the predictions of our quantitative model.

\section{ACKNOWLEDGMENT}

The authors would like to thank A. Ruiz-Columbie and R. Lopez-Boada for helpful conversations during the preparation of this manuscript.

\section{REFERENCES}

[1] C. Dragone, C. A. Edwards, and R. C. Kistler, "Integrated optics N $\times$ N multiplexer on silicon," IEEE Photon. Technol. Lett., vol. 3, no. 10, pp. 896-899, Oct. 1991.

[2] M. Smit, "New focusing and dispersive planar component based on an optical phased array," Electron. Lett., vol. 24, pp. 385-386, 1988.

[3] A. Himeno, K. Kato, and T. Miya, "Silica-based planar lightwave circuits," IEEE Sel. Topics Quantum Electron., vol. 4, no. 6, pp. 913-924, Nov./Dec. 1998.

[4] K. Okamoto, Fundamentals of Optical Waveguides. New York: Academic, 2000, pp. 341-400. 
[5] P. Muñoz, D. Pastor, and J. Capmany, "Modeling and design of arrayed waveguide gratings," J. Lightwave Technol., vol. 20, no. 4, pp. 661-674, Apr. 2002.

[6] I. Molina-Fernández and J. G. Wangüemert-Pérez, "Improved AWG Fourier optics model," Opt. Express, vol. 12, pp. 4804-4821, 2004.

[7] D. E. Leaird, A. M. Weiner, S. Shen, A. Sugita, S. Kamei, M. Ishi, and K. Okamoto, "High repetition rate femtosecond WDM pulse generation using direct space-to-time pulse shapers and arrayed waveguide gratings," Opt. Quantum Electron., vol. 33, pp. 811-826, 2001.

[8] A. Krishnan, M. Knapczyk, L. G. de Peralta, A. A. Bernussi, and H. Temkin, "Reconfigurable direct space-to-time pulse-shaper based on arrayed waveguide grating multiplexers and digital micromirrors," IEEE Photon. Technol. Lett., vol. 17, no. 9, pp. 1959-1961, Sep. 2005.

[9] J. Kani, K. Iwatsuki, and T. Imai, "Optical multiplexing technologies for access-area applications," IEEE Sel. Topics Quantum Electron., vol. 12, no. 4, pp. 661-668, Jul./Aug. 2006.

[10] A. Krishnan, L. G. de Peralta, V. Kuryatkov, A. A. Bernussi, and H. Temkin, "Direct space-to-time pulse shaper with reflective arrayed waveguide gratings and phase masks," Opt. Lett., vol. 31, pp. 640-642, 2006.

[11] A. Krishnan, L. G. de Peralta, H. Temkin, and A. A. Bernussi, "Generation of ultrafast pulse sequences with arrayed waveguide grating multiplexers subjected to modulated external stress," IEEE Photon. Technol. Lett., vol. 18, no. 10, pp. 1158-1160, May 2006.

[12] H. Yamada, H. Sanjoh, M. Kohtoku, K. Takada, and K. Okamoto, "Measurement of phase and amplitude error distributions in arrayed-waveguide grating multi/demultiplexers based on dispersive waveguide," J. Lightwave Technol., vol. 18, no. 9, pp. 1309-1320, Sep. 2000.

[13] M. E. Marhic and X. Yi, "Calculation of dispersion in arrayed waveguide grating demultiplexers by a shifting-image method," IEEE Sel. Topics Quantum Electron., vol. 8, no. 6, pp. 1149-1157, Nov./Dec. 2002.

[14] P. A. M. Dirac, The Principles of Quantum Mechanics, 4th ed. Oxford, U.K.: Oxford Univ. Press, 1967, pp. 7-14.

[15] T. Udem, R. Holzwarth, and T. W. Hänsch, "Optical frequency metrology," Nature, vol. 416, pp. 233-237, 2002.

[16] L. G. de Peralta, A. A. Bernussi, S. Frisbie, R. Gale, and H. Temkin, "Reflective arrayed waveguide grating multiplexer," IEEE Photon. Technol. Lett, vol. 15, no. 10, pp. 1398-1400, Oct. 2003.

[17] S. L. Chin, V. Francois, J. M. Watson, and C. Delisle, "Spectral modulation of two coherently separated femtosecond laser pulses," Appl. Opt., vol. 31, pp. 3383-3384, 1992.

[18] B. Rubin and R. M. Herman, "Monochromators as light stretchers," Amer. J. Phys., vol. 49, pp. 868-871, 1981

[19] C. Froehley, B. Colombeau, and M. Vampouille, "Shaping and analysis of picosecond light pulses," Prog. Opt., vol. 20, pp. 63-153, 1983.

[20] A. Siegman, Lasers. Mill Valley: University Science Books, 1986.

[21] R. P. Feynman, QED The Strange Theory of Light and Matter. Princeton, NJ: Princeton Univ. Press, 1985.
Luis Grave de Peralta received the M.S. degree in physics from Oriente University, Santiago de Cuba, Cuba, in 1982, and the Ph.D. degree in electrical engineering from Texas Tech University, Lubbock, in 2000.

He was a Professor with the Department of Experimental and Theoretical Physics, Oriente University, until 1989. His earlier work focused in VCSEL design and material characterization (luminescence and X-ray reflectivity). Since 2000, he has been involved with planar lightwave circuit design and development at Applied WDM Inc., Texas Tech University, and Multipass Corporation, Lubbock.

Ayrton A. Bernussi received the B.S., M.S., and Ph.D. degrees in physics from State University of Campinas, Campinas, Brazil, in 1981, 1984, and 1990, respectively.

In 1988, he joined the Optoelectronic Group, Brazilian Telecommunication Company (Telebras), where he was involved with the development of semiconductor lasers and materials. From 1994 to 1995 he was a Postdoctoral Researcher with the Electrical Engineering Department, Colorado State University, Fort Collins. His research during this period was on high-temperature properties of strained quantum-well lasers. During 2000-2001, he was with the National Synchrotron Light Laboratory (LNLS), Brazil, where he was involved with the development of semiconductor high-power lasers and strained nanostructures. From 2001 to 2004, he was a Research Associate with the Electrical Engineering Department, Texas Tech University, Lubbock, where he was involved with the development and characterization of planar lightwave circuits. In 2004, he joined the faculty of Texas Tech University as an Assistant Professor in the Electrical and Computer Engineering Department. His current research interests include generation of arbitrary sequences of ultrafast pulses using integrated-optic devices, and design, fabrication, and characterization of nanocavities and plasmonic waveguides.

Henryk Temkin (SM'87-F'93) has a broad record of research in semiconductors. He was a Distinguished Member of Staff in the Physics Division of Bell Laboratories, Murray Hill, NJ, from 1977 to 1992, where he was involved in research on optoelectronic devices. He was elected a Fellow of IEEE in 1988 for his contributions to the development of quantum-well lasers for optical communications. In 1992, he joined the faculty of Colorado State University, Fort Collins, as a Professor of Electrical and Computer Engineering. He moved to Texas Tech University, Lubbock, in 1996, where he serves as a Maddox Chair in Engineering. He is currently a Program Manager in the Microsystems Technology Office of DARPA. Recent research interests include wide bandgap devices, devices for wavelength-division multiplexing, and optical properties of semiconductors. He is the author and coauthor of over 400 publications and holds 27 U.S. patents. 